Article

\title{
Novel Cationic Prodrug of Ubiquinol-10 Enhances Intestinal Absorption via Efficient Formation of Nanosized Mixed-Micelles with Bile Acid Anions
}

\author{
Shuichi Setoguchi ${ }^{\dagger}$, Ryoji Hidaka ${ }^{\dagger}$, Nami Nagata-Akaho, Daisuke Watase, Mitsuhisa Koga, \\ Kazuhisa Matsunaga *, Yoshiharu Karube and Jiro Takata * \\ Faculty of Pharmaceutical Sciences, Fukuoka University, Nanakuma, Jonan-ku, Fukuoka 814-0180, Japan; \\ ssetoguchi@fukuoka-u.ac.jp (S.S.); ryozy@orange.zero.jp (R.H.); nanohana_73@jewel.ocn.ne.jp (N.N.-A.); \\ watase@fukuoka-u.ac.jp (D.W.); kogami@fukuoka-u.ac.jp (M.K.); karube@fukuoka-u.ac.jp (Y.K.) \\ * Correspondence: k-matsu@fukuoka-u.ac.jp (K.M.); jtakata@fukuoka-u.ac.jp (J.T.); \\ Tel.: +81-92-871-6631 (ext. 6672) (K.M.); +81-92-871-6631 (ext. 6662) (J.T.) \\ + These authors contributed equally to this work.
}

Academic Editor: Rafik Karaman

check for updates

Received: 30 December 2019; Accepted: 27 January 2020; Published: 27 January 2020

\begin{abstract}
The aim of this study was to develop a prodrug of ubiquinol-10 (UqH-10), the active form of ubiquinone-10 (Uq-10), for oral delivery. Bioavailability of UqH-10 is hampered by its high susceptibility to oxidation and water-insolubility. We prepared three novel $N, N$-dimethylglycine ester derivatives of $\mathrm{UqH}-10$, including a 1-monoester (UqH-1-DMG), 4-monoester (UqH-4-DMG), and 1,4-bis-ester (UqH-DMG), and assessed their physicochemical properties in vitro and in vivo. UqH-DMG spontaneously formed an aqueous micelle solution comprising $20 \mathrm{~nm}$ particles at $36.5^{\circ} \mathrm{C}$. Cationic UqH-DMG formed nano-sized $(5 \mathrm{~nm}$ ) mixed-micelles with taurocholic acid. Reconversion of the derivatives to UqH-10 was accelerated in human liver microsomes. The oral bioavailability of $\mathrm{UqH}-10$ after administration of UqH-derivatives or Uq-10 was determined in fasted and postprandial rats secreting normal and high levels of bile, respectively. In fasted rats, plasma UqH-10 after $\mathrm{UqH}$-derivatives administration reached $C_{\max }$ at $2-3 \mathrm{~h}$ and after $\mathrm{Uq}-10$ administration, it remained low. The $A U C_{0-24 \mathrm{~h}}$ of UqH-10 after UqH-derivatives administration was 2-3-fold higher than that after Uq-10 administration. In postprandial rats, the $T_{\max }$ of $\mathrm{UqH}-10$ after UqH-derivatives administration was an hour earlier than after Uq-10 administration. In conclusion, cationic UqH-derivatives are convenient prodrugs that enhance UqH-10 bioavailability by forming nanosized mixed-micelles with intestinal bile acids.
\end{abstract}

Keywords: bioavailability; coenzyme $\mathrm{Q}_{10}$; drug delivery system; prodrug; ubiquinol; ubiquinone

\section{Introduction}

Ubiquinone-10 (Uq-10), better-known as Coenzyme $\mathrm{Q}_{10}\left(\mathrm{CoQ}_{10}\right)$, is widely distributed in mammalian tissues, membranes, and cells. Uq-10 is an essential component of the mitochondrial respiratory chain, where it acts as an electron carrier and participates in ATP production. Ubiquinol-10 (UqH-10), the fully reduced form of Uq-10, acts as an antioxidant within biological membranes and in the mitochondria by either scavenging free radicals directly or by recycling other antioxidants, like $\alpha$-tocopherol and ascorbate [1,2]. Due to these beneficial roles, Uq-10 and UqH-10 are used to treat a variety of disorders, including cardiovascular syndromes and primary $\mathrm{CoQ}_{10}$-deficiency syndrome, and is available over the counter as a dietary supplement worldwide [3-6].

$\mathrm{UqH}-10$ has powerful pharmacological activities, but its bioavailability is poor due to its low water-solubility (it is highly lipophilic) and its susceptibility to rapid oxidation. A way of improving 
the bioavailability of $\mathrm{UqH}-10$ is by enhancing its diffusion efficiency in the unstirred water layer of the intestines, as this is a rate-limiting step in the intestinal absorption of lipophilic compounds [7]. Increasing aqueous solubility in the unstirred water layer is particularly important for efficient absorption of orally administered drugs. Several approaches have been tested to promote intestinal absorption, including formulating drugs in liposomes, nano-emulsions, or solid lipid nanoparticles. In particular, self-emulsified drug delivery systems (SEDDS) have been shown to induce bile secretion, leading to improved absorption [8-12]. However, these formulation techniques demand administration of large dosage volumes due to the requirement to include large amounts of fatty acids and surfactants. Smaller-volume formulations without excipients would make it easier to achieve target doses in recipients and for improved oral intake.

We hypothesized that a prodrug strategy for $\mathrm{UqH}-10$ could overcome the aforementioned poor oral bioavailability. We previously reported that $N, N$-dimethylglycine esters of vitamin $\mathrm{K}_{2}$ hydroquinone, which, similar to UqH-10, contains phenolic hydroxyl groups and a large lipophilic side chain, had good water solubility [13] and improved bioavailability over the parent drug in rats [14].

The aims of the present study were to synthesize $\mathrm{N}, \mathrm{N}$-dimethylglycine esters of UqH-10 (Figure 1), evaluate their physicochemical properties in vitro, and their pharmacokinetics after administration as a UqH-10 prodrug in vivo. Since most previous studies aimed to induce bile secretion for the absorption of Uq-10 and UqH-10, we established an in vivo model where bile secretion in rats was regulated by controlled feeding to investigate the influence of bile secretion on the absorption of the UqH-10-derivatives. We provide evidence that $\mathrm{UqH}-10 \mathrm{~N}, \mathrm{~N}$-dimethylglycine ester derivatives are prodrugs that can enhance intestinal absorption in small dosage volumes due to formation of mixed-micelles with endogenous bile acids.

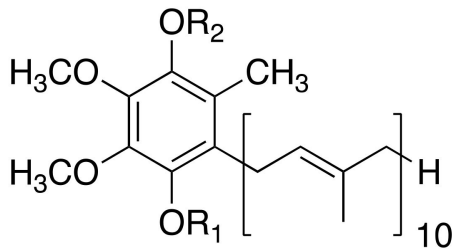

\begin{tabular}{ccc}
\hline $\begin{array}{c}\text { Ubiquinol-10 } \\
\text { derivatives }\end{array}$ & $\mathrm{R}_{1}$ & $\mathrm{R}_{2}$ \\
\hline $\begin{array}{c}\text { Ubiquinol-10 } \\
(\text { UqH-10) }\end{array}$ & $\mathrm{H}$ & $\mathrm{H}$ \\
$\begin{array}{c}\text { 1-monoester } \\
(\mathrm{UqH}-1-\mathrm{DMG})\end{array}$ & $-\mathrm{COCH}_{2} \mathrm{~N}\left(\mathrm{CH}_{3}\right)_{2} \cdot \mathrm{HCl}$ & $\mathrm{H}$ \\
$\begin{array}{c}\text { 4-monoester } \\
(\mathrm{UqH}-4-\mathrm{DMG})\end{array}$ & $\mathrm{H}$ & $-\mathrm{COCH}_{2} \mathrm{~N}\left(\mathrm{CH}_{3}\right)_{2} \cdot \mathrm{HCl}$ \\
$\begin{array}{c}\text { 1,4-bis-ester } \\
(\mathrm{UqH}-\mathrm{DMG})\end{array}$ & $-\mathrm{COCH}_{2} \mathrm{~N}\left(\mathrm{CH}_{3}\right)_{2} \cdot \mathrm{HCl}$ & $-\mathrm{COCH}_{2} \mathrm{~N}\left(\mathrm{CH}_{3}\right)_{2} \cdot \mathrm{HCl}$ \\
\hline
\end{tabular}<smiles>COC1=C(OC)C(=O)C(C(C)(C=CC=O)CC=O)=C(C)C1=O</smiles>

Ubiquinone-10 (Uq-10)

Figure 1. Chemical structures of Ubiquinol-10 derivatives and Ubiquinone-10.

\section{Results and Discussion}

\subsection{Physicochemical Properties of UqH-10 Derivatives}

Two types of monoester and a bis-ester were successfully obtained by chemical synthesis (Figure 1) and characterized by MS, elemental analysis, and ${ }^{1} \mathrm{H}-{ }^{13} \mathrm{C}$ HMBC NMR. The melting points of the monoesters (UqH-1-DMG, UqH-4-DMG) and the bis-ester (UqH-DMG) were approximately $80^{\circ} \mathrm{C}$ and $180^{\circ} \mathrm{C}$, respectively (shown in Section 3.4). The melting points of these esters were much higher than that of Uq-10 $\left(48^{\circ} \mathrm{C}\right)$. UqH-DMG formed a transparent solution in water at concentrations up to $5 \mathrm{mM}$. 
Its water solubility was significantly higher than that of Uq-10, which was "practically insoluble, or insoluble" (even when diluted to $<0.116 \mathrm{mM}$ ) based on the Japanese Pharmacopoeia monograph. Both monoesters and the bis-ester were stable against oxidation under atmospheric conditions.

\subsection{Micelle Formation of UqH-DMG in Water}

To investigate the influence of temperature on the solubility of UqH-DMG, visual inspections of the solutions were performed in test tubes. A $45 \mathrm{mM}$ of UqH-DMG dissolved in water appeared opaque at $27.0^{\circ} \mathrm{C}$, but after increasing the temperature to $36.5^{\circ} \mathrm{C}$, the solution became transparent, indicating that UqH-DMG formed a micelle solution (Figure 2A). The micelle size was confirmed in Section 2.3 below (Figure 3B). The solubilizing points at different concentrations of UqH-DMG were also measured and plotted (Figure 2B). An exponential increase in solubilizing point was observed immediately above $45 \mathrm{mM}$ UqH-DMG, indicating that the Krafft's point of UqH-DMG, a cationic large molecule, in water is around $36.5^{\circ} \mathrm{C}$. Therefore, it is likely that UqH-DMG in aqueous medium would be soluble at body temperature $\left(\sim 37^{\circ} \mathrm{C}\right)$ after oral administration.

A

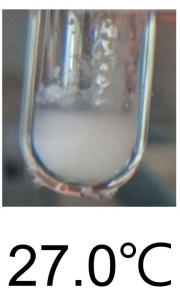

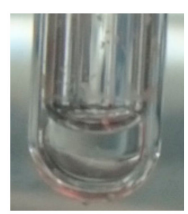

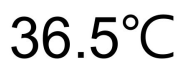

B

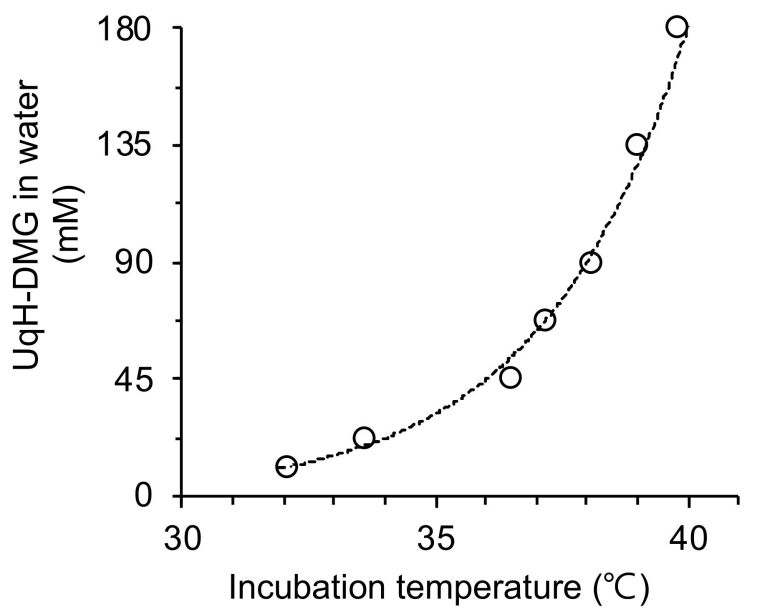

Figure 2. Influence of incubation temperatures on the solubility of UqH-DMG. (A) Representative photographs of $45 \mathrm{mM}$ of UqH-DMG in water at $27.0^{\circ} \mathrm{C}$ and $36.5^{\circ} \mathrm{C}$. (B) Solubilizing points of $\mathrm{UqH}-\mathrm{DMG}$ in water versus incubation temperatures.

Water solubility of UqH monoesters (UqH-1-DMG and UqH-4-DMG) is lower than that of UqH bis-ester (UqH-DMG). Related Krafft's points of the monoesters were not observed, since the effect of ionic surfactant of $\mathrm{UqH}$ monoesters were not observed due to the differences in the balance of polar group(s) and hydrophobic group between UqH bis-ester and monoesters.

\subsection{Mixed-Micelle Formation of UqH-DMG with Taurocholic Acid}

After UqH-DMG is solubilized in the stomach under low $\mathrm{pH}$, it is expected that UqH-DMG will encounter bile acid anions in the intestinal lumen under neutral $\mathrm{pH}$. Therefore, the interaction between UqH-DMG and taurocholic acid (TCA), a representative bile acid, was investigated. The appearance of a mixture of UqH-DMG:TCA at a molar ratio of 1: 0.5 (Figure 3A) was slightly cloudy, but at higher proportions of TCA, i.e., UqH-DMG:TCA ratios of 1:1 to 1:10, the solutions became transparent (Figure 3A). Solutions of $20 \mathrm{mM} \mathrm{UqH}-\mathrm{DMG}$ in water and a mixture of UqH-DMG:TCA at a 1:1 molar ratio were subjected to dynamic light scattering measurements. Consistent with the appearances of the solutions shown in Figure 3A, the Z-average particle diameter of the $20 \mathrm{mM}$ UqH-DMG solution was $20.38 \pm 0.14$ (mean $\pm \mathrm{SD}$ ) $\mathrm{nm}$, higher than that of the UqH-DMG/TCA mixture, which was 5.183 \pm 0.033 (mean $\pm \mathrm{SD}$ ) $\mathrm{nm}$. These results suggest that the UqH-DMG: TCA mixture at a 1:0.5 molar 
ratio was cloudy because of complex formation, and at higher molar concentrations of TCA relative to UqH-DMG, mixed-micelles particles of approximately $5 \mathrm{~nm}$ in diameter form spontaneously. Small micelle particles under $10 \mathrm{~nm}$ in diameter are expected to diffuse efficiently in the unstirred water layer of the intestines, as this is a rate-limiting step in the intestinal absorption.

A

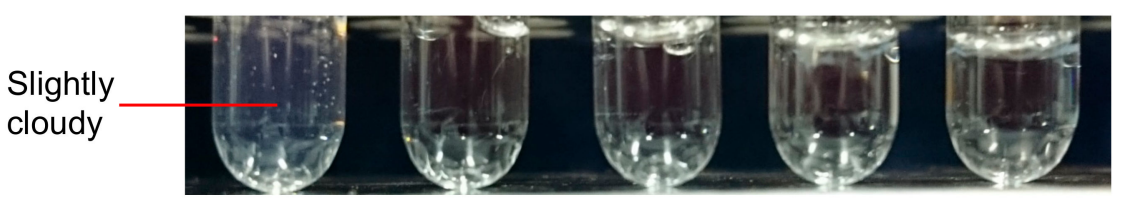

\begin{tabular}{lccccc}
\hline \multirow{2}{*}{ Contents } & \multicolumn{5}{c}{ Molar ratio } \\
\cline { 2 - 6 } & $\mathrm{a}$ & $\mathrm{b}$ & $\mathrm{c}$ & $\mathrm{d}$ & $\mathrm{e}$ \\
\hline UqH-DMG & 1 & 1 & 1 & 1 & 1 \\
TCA & 0.5 & 1 & 2.5 & 5 & 10 \\
\hline
\end{tabular}

B

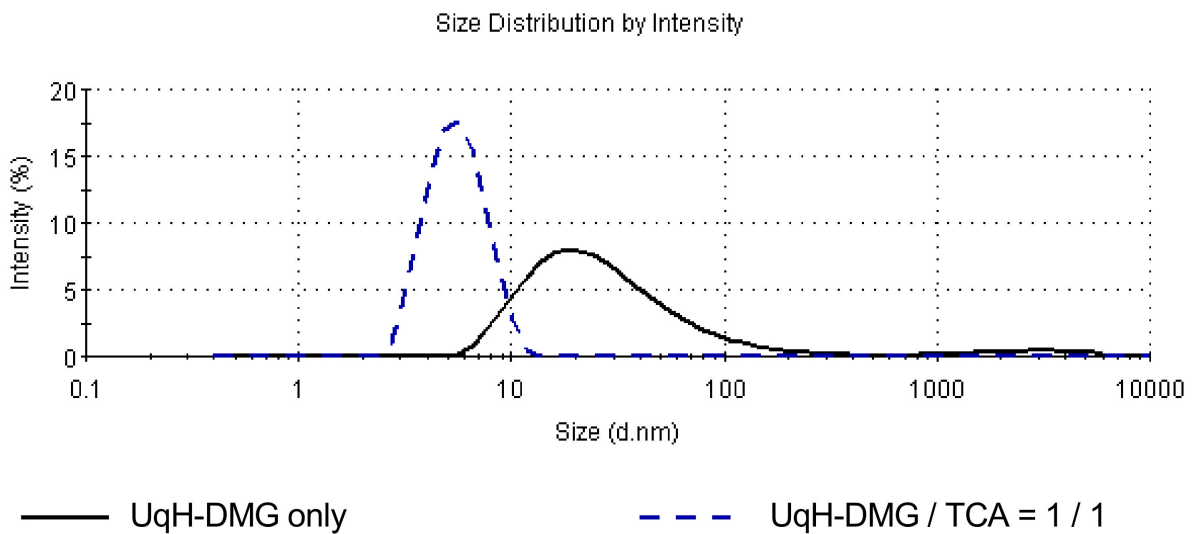

Figure 3. Effect of taurocholic acid on UqH-DMG solubility. (A) Appearance of mixtures of UqH-DMG and taurocholic acid (TCA) at different molar ratios. (B) Particle size distribution of UqH-DMG with or without TCA. Solid line: UqH-DMG only, dashed line: UqH-DMG:TCA at a molar ratio of 1:1.

For the interactions between UqH monoesters and taurocholic acid, UqH-4-DMG was solubilized with, a 10-times molar-equivalents, taurocholic acid. A typical mixed-micelle is the state at which an anion-cation ion pair(s) is solubilized in either richer component. The types of micelle formed with taurocholic acid may differ between $\mathrm{UqH}$ bis-ester and monoesters.

\subsection{Enzymatic Hydrolysis of $U q H-10$ Derivatives}

To determine whether UqH-derivatives can be activated by liver esterases to revert to their parent form (UqH-10), their hydrolysis in rat and human liver microsomes was assessed. The UqH-DMG (bis-ester) is expected to be hydrolyzed in a sequential reaction via either 1-mono or 4-monoester, as previously reported for vitamin $\mathrm{K}$ hydroquinone [13]. Hence, the hydrolysis velocities of UqH-monoesters were compared. The velocity versus concentration profile was fitted using the Michaelis-Menten model, and the kinetic parameters were calculated using GraphPad Prism 6 (GraphPad Software, CA, USA) (Table 1). In rat liver microsomes, hydrolysis of UqH-4-DMG occurred much faster than UqH-1-DMG. The $\mathrm{V}_{\text {max }} / \mathrm{K}_{\mathrm{m}}$ value of UqH-4-DMG was 8.9-fold higher than that of UqH-1-DMG. The hydrolysis of UqH-DMG was also assessed by the rate of generation of 
UqH-10. The $\mathrm{K}_{\mathrm{m}}$ and $\mathrm{V}_{\text {max }}$ parameters were similar to those of UqH-1-DMG. Therefore, hydrolysis of UqH-DMG at position 1 is the rate-limiting step for conversion to UqH-10. In other words, the rates of hydrolysis depend on the esterified position on UqH-10. To simplify comparisons between experiments in rat and human microsomes, only UqH-4-DMG was subjected to a human microsome hydrolysis assay. UqH-4-DMG was readily cleaved in human liver microsomes, and the $\mathrm{V}_{\max }$ and $\mathrm{K}_{\mathrm{m}}$ values of UqH-4-DMG hydrolysis were similar to those observed in rat liver microsomes. Furthermore, to determine whether the hydrolysis of UqH-4-DMG was esterase-dependent, an esterase inhibitor, eserine, was added to the reactions. As shown in Supplemental Figure S1, eserine inhibited UqH-4-DMG hydrolysis in both rat and human liver microsomes in a concentration-dependent manner. These results strongly suggest that $\mathrm{UqH}$-derivatives will be activated by the esterases in human livers.

Table 1. Kinetic parameters for hydrolysis of UqH-DMG, UqH-1-DMG and UqH-4-DMG in rat and human liver microsomes at $\mathrm{pH} 7.4$ and $37^{\circ} \mathrm{C}$.

\begin{tabular}{|c|c|c|c|c|}
\hline \multirow{2}{*}{ Parameters } & $\mathrm{UqH}^{-D M G}{ }^{\mathrm{a}}$ & UqH-1-DMG & \multicolumn{2}{|c|}{ UqH-4-DMG } \\
\hline & & Rat & & Human \\
\hline $\begin{array}{c}K_{\mathrm{m}} \\
\left(\times 10^{-3} \mathrm{M}\right)\end{array}$ & $3.89^{\mathrm{a}}$ & 4.63 & 0.0751 & 0.0722 \\
\hline $\begin{array}{c}V_{\max } \\
\left(\times 10^{-6} \mathrm{M} \cdot \min ^{-1}\right)\end{array}$ & $0.603^{a}$ & 0.821 & 0.119 & 0.123 \\
\hline $\begin{array}{c}V_{\max } / K_{\mathrm{m}} \\
\left(\times 10^{-3} \mathrm{~min}^{-1}\right)\end{array}$ & $0.155^{\mathrm{a}}$ & 0.177 & 1.58 & 1.70 \\
\hline
\end{tabular}

The values are obtained from Michaelis-Menten curve fitting (GraphPad Prism). ${ }^{\text {a }}$ The kinetic parameters were calculated using the generated $\mathrm{UqH}-10$ levels after a sequential hydrolysis process.

\subsection{Plasma Concentrations of UqH-10 after Single Oral Administration of UqH-Derivatives in Fasted and Postprandial Rats}

To establish the utility of UqH-derivatives as prodrugs, we examined the plasma kinetics of UqH-10 after single oral administration of UqH-4-DMG and UqH-DMG in fasted rats. Based on preliminary tests, approximately $70 \%$ of total $\mathrm{Uq}-10$ (Uq-10 and $\mathrm{UqH}-10$ ) existed in the reduced form, UqH-10. However, UqH-10 was partly oxidized during HPLC sample preparation. Therefore, the total amount of $\mathrm{UqH}-10$ was taken to be the sum of $\mathrm{UqH}-10$ and $\mathrm{Uq}-10$. Plasma concentrations versus time plots from rats after oral administration of Uq-10, UqH-4-DMG, or UqH-DMG are shown in Figure 4A. The pharmacokinetic parameters for $\mathrm{UqH}-10$ were determined by moment analysis and summarized in Table 2. The $\mathrm{C}_{\max }, \mathrm{T}_{\max }, \mathrm{AUC}$, and MRT parameters were standardized based on control levels of $\mathrm{UqH}-10$ at each time point that were individually obtained from control rats. Plasma UqH-10 levels after the administration of $\mathrm{UqH}-4-\mathrm{DMG}$ and $\mathrm{UqH}-\mathrm{DMG}$ were significantly elevated and reached $\mathrm{C}_{\max }$ at 2 and $3 \mathrm{~h}$, respectively. These results demonstrate that UqH-derivatives reverted to the parent form (UqH-10) in rats and acted as UqH-10-prodrugs. In contrast, plasma UqH-10 levels after Uq-10 administration remained low at approximately $0.2 \mu \mathrm{M}$. $\mathrm{AUC}_{0-24 \mathrm{~h}}$ values of $\mathrm{UqH}-10$ after UqH-4-DMG and $\mathrm{UqH}$-DMG administration were 3.30- and 2.36-fold higher than that after Uq-10 administration, respectively. These results indicate that UqH-prodrugs can be solubilized by low bile acid levels and efficiently absorbed in rat intestines. 
A

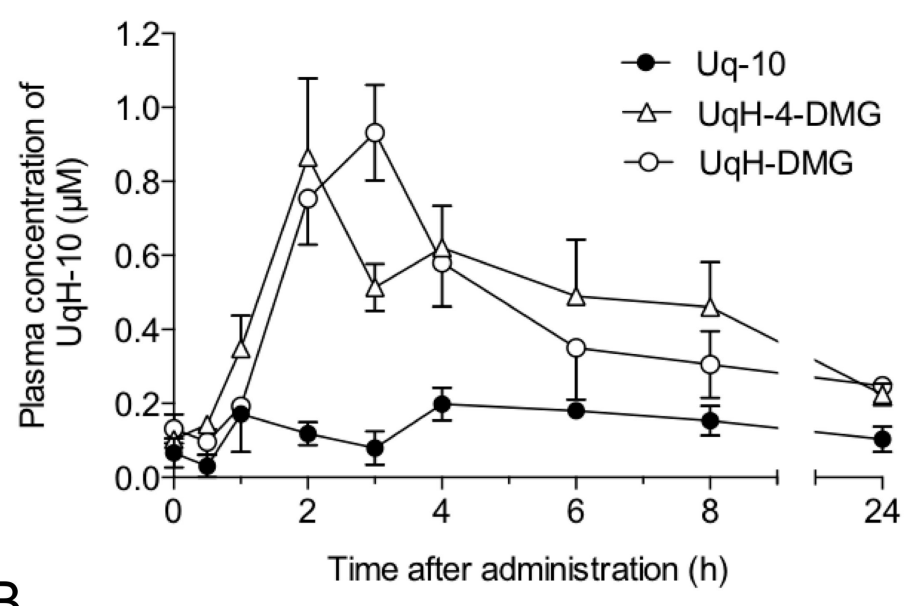

B

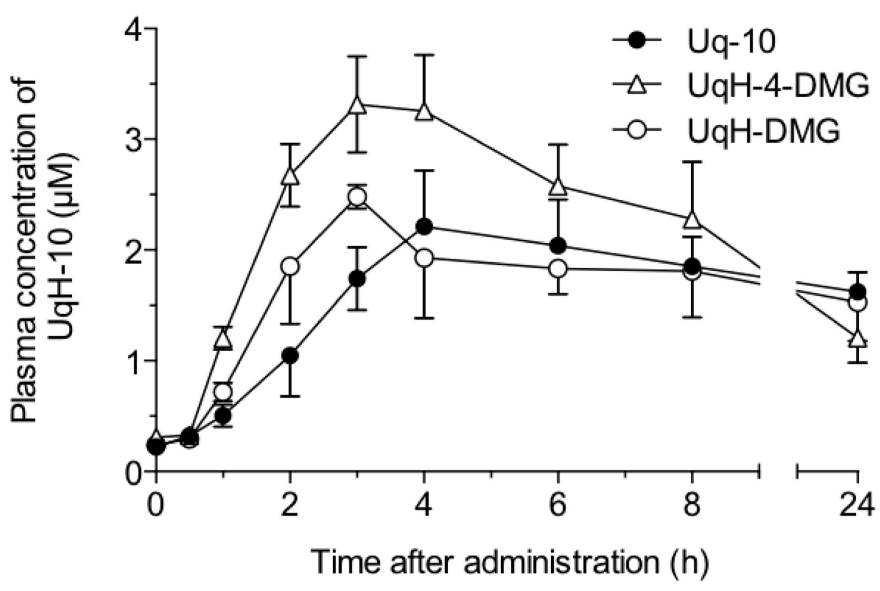

Figure 4. Plasma concentration of $\mathrm{UqH}-10$ after administration of $\mathrm{UqH}$-derivatives or $\mathrm{Uq}-10$ in fasted and postprandial rats. (A) Plasma concentration of UqH-10 after administration of $40.5 \mathrm{mmol} / \mathrm{kg}$ weight of UqH-DMG, UqH-4-DMG or Uq-10 in fasted rats (UqH-DMG, $\mathrm{n}=6$; UqH-4-DMG, $\mathrm{n}=5$; Uq-10, $\mathrm{n}=4$ ); (B) plasma concentration of UqH-10 after administration of $40.5 \mathrm{mmol} / \mathrm{kg}$ weight of $\mathrm{UqH}-\mathrm{DMG}, \mathrm{UqH}-4-\mathrm{DMG}$ or Uq-10 in postprandial state rats $(\mathrm{n}=3)$. The values indicate mean $\pm \mathrm{SE}$.

Table 2. Pharmacokinetic parameters for UqH-10 after oral administration of UqH-DMG, UqH-4-DMG, or Uq-10 in fasted and postprandial rats.

\begin{tabular}{|c|c|c|c|c|c|c|}
\hline Parameters & Uq-10 & UqH-4-DMG & UqH-DMG & Uq-10 & UqH-4-DMG & UqH-DMG \\
\hline & & Fasted & & & Postprandial & \\
\hline$n$ & 4 & 5 & 6 & 3 & 3 & 3 \\
\hline Dose $\left(\mu \mathrm{mol} \cdot \mathrm{kg}^{-1}\right)$ & & & 40.5 & & & \\
\hline$C_{\max }\left(\mu \mathrm{mol} \cdot \mathrm{L}^{-1}\right)$ & $0.143 \pm 0.0826$ & $0.763 \pm 0.209$ & $0.800 \pm 0.157$ & $1.99 \pm 0.472$ & $3.01 \pm 0.423$ & $2.24 \pm 0.110$ \\
\hline$T_{\max }(\mathrm{h})$ & 1 & 2 & 3 & 4 & 3 & 3 \\
\hline $\begin{array}{c}A U C_{0-24 \mathrm{~h}} \\
\left(\mu \mathrm{mol} \cdot \mathrm{L}^{-1} \cdot \mathrm{h}\right)\end{array}$ & $2.16 \pm 0.878$ & $7.13 \pm 1.71$ & $5.10 \pm 1.57$ & $35.0 \pm 1.78$ & $39.3 \pm 6.78$ & $34.4 \pm 6.51$ \\
\hline$M R T(\mathrm{~h})$ & $8.56 \pm 1.96$ & $8.96 \pm 0.710$ & $8.43 \pm 0.956$ & $12.2 \pm 0.971$ & $9.12 \pm 0.565$ & $11.5 \pm 0.525$ \\
\hline
\end{tabular}

Values are each the mean \pm SE. The calculated parameters, $C_{\max }, T_{\max }, A U C$ and MRT, were standardized by base levels of plasma UqH-10 at each point obtained from control rats.

To determine the effects of bile secretion on the intestinal absorption of UqH-prodrugs, we established a postprandial state model to enrich bile secretion in rats. The rats were fed for only $2 \mathrm{~h}$ each day during the three weeks prior to administration of UqH-prodrugs and Uq-10. Plasma levels of UqH-10 after single oral administration of UqH-prodrugs and Uq-10 in the postprandial rats were significantly higher than those in fasted rats (Figure $4 \mathrm{~A}, \mathrm{~B}$ ). Even after the $\mathrm{T}_{\max }$, plasma levels of $\mathrm{UqH}-10$ in postprandial rats were maintained around $2 \mu \mathrm{M}$. These results suggest that abundant 
bile secretion induced by controlled feeding strongly promotes the solubilization of UqH-prodrugs and Uq-10. Although there are little differences in the $\mathrm{AUC}_{0-24 \mathrm{~h}}$ values of $\mathrm{UqH}-10$ between $\mathrm{Uq}-10$ and UqH-prodrugs administrations under abundant bile secretion conditions, the UqH-prodrugs brought forward the $\mathrm{T}_{\max }$ of plasma $\mathrm{UqH}$ to an hour earlier than after Uq-10 administration. The change in $\mathrm{T}_{\max }$ suggests that $\mathrm{UqH}$-prodrugs could solubilize into mixed-micelles upon encounter of bile acid anions, and did so more efficiently than Uq-10. The results from fasted and postprandial rats show that Uq-10 is susceptible to intestinal bile levels but UqH-prodrugs are not.

An innovative self-emulsified drug delivery system (SEDDS) for Uq-10 was previously proposed by Onoue et al [9]. They reported that an SEDDS formulation of Uq-10 improved the oral bioavailability of Uq-10 compared to crystalline Uq-10. However, this formulation technique tended to produce larger dosage volumes because it required an equal amount of fatty acid triglycerides and 8-fold more surfactant relative to Uq-10. In contrast, the current prodrug strategy for $\mathrm{UqH}-10$ can be used to formulate smaller dosage volumes as they form very small particles $(\sim 5 \mathrm{~nm})$ with endogenous bile acid. This is an improved procedure for developing suitable dosage formulations for easier oral uptake and enhanced intestinal diffusion.

In conclusion, UqH-4-DMG and UqH-DMG may be good prodrug candidates with enhanced intestinal absorption due to their ability to form mixed-micelles with bile acid anions. Additional screening tests to identify the best formulation of UqH-prodrugs, and additional pharmacokinetic studies will help inform future development of this important biomolecule for health and medical applications.

\section{Materials and Methods}

\subsection{Chemicals}

Uq-10 was a generous gift from Kaneka Corporation (Osaka, Japan) and was used as received. $\mathrm{N}, \mathrm{N}$-Dimethyl glycine hydrochloride was from Tokyo Kasei Kogyo Co., Ltd. (Tokyo, Japan). Eserine (physostigmine sulfate) was from Sigma-Aldrich Chemical Co. (St. Louis, MO, USA). All other chemicals were from FUJIFILM Wako Pure Chemical Corporation (Osaka, Japan).

\subsection{Animals}

Male Sprague-Dawley (SD) rats (eight weeks old), SD rat liver microsomes, and human liver microsomes were obtained from Charles River, Japan, Inc. (Kanagawa, Japan). All animal care and use procedures were performed in compliance with the regulations established by the Experimental Animal Care and Use Committee of Fukuoka University, which are in accordance with the universal principles of laboratory animal care.

\subsection{Instrumental Analyses}

All melting points were determined with a BY-1 micro melting point apparatus (Yazawa, Tokyo, Japan) and were uncorrected. Elementary analyses, ${ }^{1} \mathrm{H}-\mathrm{NMR}$ and mass spectra measurements were performed at the Central Microanalytical Department of Pharmaceutical Sciences, Fukuoka University. The ${ }^{1} \mathrm{H}-\mathrm{NMR}$ spectra were determined at $500 \mathrm{MHz}$ in $\mathrm{CDCl}_{3}$ using a JEOL GX-500b spectrometer (JEOL Ltd, Tokyo, Japan). The chemical shifts are expressed in $\delta(\mathrm{ppm})$, using tetramethylsilane as the internal standard, with the following abbreviations: $\mathrm{s}=$ singlet, $\mathrm{d}=$ doublet, $\mathrm{m}=$ multiplet. The coupling constant J was measured in Hz. fast atom bombardment mass (FAB-MS) spectra were obtained using a JEOL DX-300 spectrometer (JEOL Ltd, Tokyo, Japan). The ${ }^{1} \mathrm{H}-\mathrm{NMR}$ and MS spectra of UqH-derivatives are shown in the Supplemental Figures S2 and S3, respectively.

\subsection{Synthesis of Ubiquinol-10 N,N-Dimethylglycinate Derivatives}

Uq-10 (3.475 mmol) was dissolved in $150 \mathrm{~mL}$ distilled isopropyl ether. Sodium tetrahydroborate (9.91 mmol), suspended in methanol, was added to this ether solution and stirred for $3 \mathrm{~min}$ in the dark. 
After reduction of Uq-10 was completed, the mixture was treated with $100 \mathrm{~mL}$ degassed water and extracted with distilled isopropyl ether. The organic layer was dried over anhydrous sodium sulfate and evaporated in vacuo. The resulting residue, a milk-white solid, was used in the following steps as UqH-10.

$N$,N-Dimethylglycine (10.424 mmol) and dicyclohexylcarbodiimide (DCC, $10.424 \mathrm{mmol})$ in 20 $\mathrm{mL}$ of dry pyridine were stirred for $20 \mathrm{~min}$ and then UqH-10 (3.475 mmol) was added. The reaction mixture was stirred for $14 \mathrm{~h}$ at room temperature in the dark.

After the reaction, the dicyclohexylurea formed was removed by filtration, and the filtrate was evaporated in vacuo. The residue was suspended in $100 \mathrm{~mL}$ distilled water and alkalinized with sodium bicarbonate. Dicyclohexylurea was completely removed by filtration and the filtrate was treated with $150 \mathrm{~mL}$ water and made alkaline with sodium bicarbonate. Then the solution was extracted with ethyl acetate. The organic layer was dried over anhydrous sodium sulfate and evaporated. The residue was fractionated on Flash 40+M silica gel columns $(\varphi 40 \times 150$ mm, Biotage Japan Co., Ltd., Tokyo, Japan), eluted with a gradient of 1:1 to 2:8 $(v / v)$ n-hexane/ethyl acetate. This yielded two fractions, a mixture of monoesters $\left(R_{\mathrm{f}}=0.45\right)$ and the 1,4 -bis ester $\left(\mathrm{R}_{\mathrm{f}}=0.36\right)$. $n$-Hexane containing $3 \mathrm{~N}$ dioxane hydrochloride was added to the 1,4-bis ester fraction and the precipitate was collected and recrystallized from acetone to give the hydrochloride salt of 2,3-dimethoxy-5-methyl-6-decaprenyl-benzene-1,4-bis- $N, N$-dimethyl glycinate (UqH-DMG). The compound was a white solid.

UqH-DMG: Yield 42\%. mp 179-181 ${ }^{\circ} \mathrm{C} .{ }^{1} \mathrm{H}-\mathrm{NMR}\left(500 \mathrm{MHz}, \mathrm{CDCl}_{3}\right) \delta$ : Uq-10 moiety; $1.55-1.62$ $\left(\mathrm{CH}_{3} \times 9, \mathrm{~m}\right), 1.68\left(\mathrm{CH}_{3}, \mathrm{~d}, J=1.0\right), 1.72\left(\mathrm{CH}_{3}, \mathrm{~d}, J=1.0\right), 1.94-2.16\left(\mathrm{CH}_{2} \times 18, \mathrm{~m}\right), 2.08\left(\mathrm{CH}_{3}, \mathrm{~s}\right), 3.22$ $\left(\mathrm{CH}_{2}, \mathrm{~d}, \mathrm{~J}=6.5\right), 3.84\left(\mathrm{CH}_{3} \times 2, \mathrm{~s}\right), 4.88-5.13(\mathrm{CH} \times 10, \mathrm{~m}), \mathrm{N}, \mathrm{N}$-dimethylglycine moiety; $3.07\left(\mathrm{CH}_{3} \times\right.$ 2, s), $3.10\left(\mathrm{CH}_{3} \times 2, \mathrm{~s}\right), 4.21\left(\mathrm{CH}_{2}, \mathrm{~s}\right), 4.31\left(\mathrm{CH}_{2}, \mathrm{~s}\right)$. FAB-MS $(\mathrm{m} / \mathrm{z}) ; 1035\left(\mathrm{M}^{+}-2 \mathrm{HCl}\right)$. Anal. Calcd for $\mathrm{C}_{67} \mathrm{H}_{108} \mathrm{~N}_{2} \mathrm{O}_{6} \mathrm{Cl}_{2}$ : C, 72.60; H, 9.82; N, 2.53. Found: C, 72.36; H, 9.94; N, 2.42.

The mixture of monoesters was fractionated by HPLC. A normal phase column Mightysil Si60 $(\varphi 20 \times 250 \mathrm{~mm}$, Kanto Chemical, Tokyo, Japan) and a mobile phase of n-hexane-ethyl acetate (7:3, $v / v$ ) at a flow rate of $15 \mathrm{~mL} / \mathrm{min}$, was used. The eluent was detected spectrophotometrically at 280 $\mathrm{nm}$. The retention times for the 1-monoester and 4-monoester were 25.7 and $33.0 \mathrm{~min}$, respectively. Corresponding fractions were collected in $\mathrm{n}$-hexane containing $3 \mathrm{~N}$ dioxane hydrochloride and isolated as the hydrochloride salts of the 1-monoester (UqH-1-DMG) and 4-monoester (UqH-4-DMG). Both were white solids. The structures of UqH-1-DMG and UqH-4-DMG were determined by using ${ }^{1} \mathrm{H}_{-}{ }^{13} \mathrm{C}$ HMBC NMR techniques.

UqH-1-DMG: Yield 19\%. mp 82-85 ${ }^{\circ} \mathrm{C} .{ }^{1} \mathrm{H}-\mathrm{NMR}\left(500 \mathrm{MHz}, \mathrm{CDCl}_{3}\right)$ 8: Uq-10 moiety; 1.57-1.62 $\left(\mathrm{CH}_{3} \times 9, \mathrm{~m}\right), 1.67\left(\mathrm{CH}_{3}, \mathrm{~d}, J=1.0\right), 1.71\left(\mathrm{CH}_{3}, \mathrm{~d}, J=1.0\right), 1.57-1.62\left(\mathrm{CH}_{2} \times 18, \mathrm{~m}\right), 2.14\left(\mathrm{CH}_{3}, \mathrm{~s}\right), 3.17$ $\left(\mathrm{CH}_{2}, \mathrm{~d}, \mathrm{~J}=7.0\right), 3.82\left(\mathrm{CH}_{3}, \mathrm{~s}\right), 3.90\left(\mathrm{CH}_{3}, \mathrm{~s}\right), 4.90-5.12(\mathrm{CH} \times 10, \mathrm{~m}), \mathrm{N}, \mathrm{N}$-dimethylglycine moiety; 3.05 $\left(\mathrm{CH}_{3} \times 2, \mathrm{~s}\right), 4.10\left(\mathrm{CH}_{2}\right.$, s). FAB-MS $(\mathrm{m} / \mathrm{z}) ; 950\left(\mathrm{M}^{+}-\mathrm{HCl}\right)$. Anal. Calcd for $\mathrm{C}_{63} \mathrm{H}_{100} \mathrm{NO}_{5} \mathrm{Cl}+0.5 \mathrm{H}_{2} \mathrm{O}: \mathrm{C}$, 75.98; H, 10.22; N, 1.41. Found: C, 75.89; H, 10.30; N, 1.39.

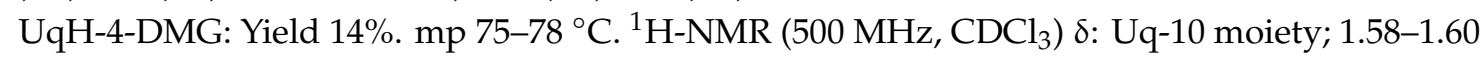
$\left(\mathrm{CH}_{3} \times 9, \mathrm{~m}\right), 1.68\left(\mathrm{CH}_{3}, \mathrm{~d}, J=1.0\right), 1.76\left(\mathrm{CH}_{3}, \mathrm{~d}, J=1.0\right), 1.98-2.09\left(\mathrm{CH}_{2} \times 18, \mathrm{~m}\right), 2.03\left(\mathrm{CH}_{3}, \mathrm{~s}\right), 3.35$ $\left(\mathrm{CH}_{2}, \mathrm{~d}, \mathrm{~J}=7.0\right), 3.82\left(\mathrm{CH}_{3}, \mathrm{~s}\right), 3.90\left(\mathrm{CH}_{3}, \mathrm{~s}\right), 5.07-5.13(\mathrm{CH} \times 10, \mathrm{~m}), \mathrm{N}, \mathrm{N}$-dimethylglycine moiety; 3.08 $\left(\mathrm{CH}_{3} \times 2, \mathrm{~s}\right), 4.17\left(\mathrm{CH}_{2}, \mathrm{~s}\right)$. FAB-MS $(\mathrm{m} / \mathrm{z}) ; 950\left(\mathrm{M}^{+}-\mathrm{HCl}\right)$. Anal. Calcd for $\mathrm{C}_{63} \mathrm{H}_{100} \mathrm{NO}_{5} \mathrm{Cl}+\mathrm{H}_{2} \mathrm{O}: \mathrm{C}$, 75.30; H, 10.23; N, 1.39. Found: C, 75.30; H, 10.47; N, 1.39 .

\subsection{Water Solubility}

The aqueous solubility of each ester was determined by adding $20 \mu \mathrm{mol}$ of each compound to 1 $\mathrm{mL}$ distilled water in amber test tubes and incubating the tubes at $25 \pm 1{ }^{\circ} \mathrm{C}$ in a constant-temperature water bath. The test tubes were shaken for $24 \mathrm{~h}$ and the suspensions were centrifuged at $1250 \times g$ for $10 \mathrm{~min}$. The supernatants were diluted 10-fold with distilled water and assayed by the HPLC method described in Section 3.9.1. 


\subsection{Micellization of UqH-DMG in Water}

UqH-DMG was diluted to intended concentrations with milliQ water in glass tubes. The test tubes were incubated in a water bath equipped with a thermometer. The solution appearance was visually inspected and the solubilizing points were plotted on a concentration versus incubation temperature curve.

\subsection{Mixed-Micellization of UqH-DMG with Taurocholic Acid}

\subsubsection{Preparation of Aqueous Solutions of UqH-DMG with Taurocholic Acid}

A solution of $20 \mathrm{mM} \mathrm{UqH}-\mathrm{DMG}$ was prepared in distilled water and incubated at $36.5^{\circ} \mathrm{C}$ until the solution was translucent. The solution was combined with $10 \mathrm{mM}$ TCA aqueous solution in the final molar ratios of 1:0.5-10 UqH-DMG:TCA.

\subsubsection{Determination of Particle Sizes}

The Z-average of diameters of the particles of aqueous solution of UqH-DMG prepared in a polystyrene cuvette in Section 3.7.1. above were determined by a Zetasizer Nano ZS (MALVERN, Worcestershire, UK). The measurements were performed three independent times for each sample.

\subsection{Enzymatic Hydrolysis of UqH-Derivatives}

The hydrolysis of esters was analyzed at $37{ }^{\circ} \mathrm{C}$ in phosphate buffered saline (PBS) containing commercially available rat or human liver microsomes (In Vitro Technologies, Inc., Baltimore, MD, USA). The microsomes $(20 \mathrm{mg} / \mathrm{mL})$ were adjusted to a protein concentration of $2 \mathrm{mg} / \mathrm{mL}$ and were preincubated at $37^{\circ} \mathrm{C}$ for $5 \mathrm{~min}$ before adding the esters.

Stock solutions of esters were prepared in ethanol. The enzymatic reactions were initiated by adding $10 \mu \mathrm{L}$ ester stock solution (final concentration $0.1-0.4 \mathrm{mM}$ ) and $50 \mu \mathrm{L}$ PBS to $940 \mu \mathrm{L}$ of preheated reaction medium containing rat or human liver microsomes in amber test tubes. These reactions were incubated at $37^{\circ} \mathrm{C}$ and, at various times, $100 \mu \mathrm{L}$ aliquots were removed and mixed with 100 $\mu \mathrm{L} 10 \%$ trichloroacetic acid. Samples were then vortex mixed for 2 min and $800 \mu \mathrm{L}$ ethyl acetate was added, with vortex mixing for $5 \mathrm{~min}$. The samples were centrifuged at $1250 \times g$ for $10 \mathrm{~min}$. The $20 \mu \mathrm{L}$ organic layer was analyzed by the HPLC system described in Section 3.9.1. The initial hydrolytic rate (mM UqH-10 formed per min) was calculated from the initial slope of a plot of UqH-10 concentration versus time.

Effects of eserine on UqH-4-DMG hydrolysis in liver microsomes was also examined, using similar methods, with $50 \mu \mathrm{L}$ eserine aqueous solution added, instead of PBS, to the mixtures with liver microsomes. Eserine was tested at 0-2.0 $\mathrm{mM}$.

\subsection{HPLC Analysis}

\subsubsection{HPLC System and Conditions for Water Solubility and Hydrolysis Study}

The Shimadzu HPLC system (Shimadzu, Kyoto, Japan) consisted of a pump (LC-10AD), an autoinjector (SIL-10ADvp), a UV detector (SPD-10Avp), and a column oven (CTO-10Avp). The eluent was monitored spectrophotometrically at $280 \mathrm{~nm}$. Compounds were analyzed on a YMC-Pack C8 reversed-phase column $(\varphi 4.6 \times 250 \mathrm{~mm}$, YMC Co., Ltd., Kyoto, Japan) protected by a $\mu$ Bondapak C18 Guard-Pak (Waters Co., Milford, MA, USA) with a degassed mobile phase of methanol and 2-propanol $(850 / 150, v / v)$ at $0.8 \mathrm{~mL} / \mathrm{min}$. UqH-10 and Uq-10 were quantified using linear calibration curves of peak areas versus concentrations. UqH-10 was partly oxidized during the preparation procedure. Therefore, the sum of UqH-10 and Uq-10 represented total UqH-10. Retention times were: UqH-10, 11.6 min; UqH-ester derivatives, $13.2 \mathrm{~min}$ and Uq-10, $15.4 \mathrm{~min}$. 


\subsubsection{HPLC System and Conditions for Pharmacokinetic Study}

The HPLC system consisted of a pump (Model LC-10AD, Shimadzu), an autoinjector (Model SIL-10ADvp, Shimadzu) and a column oven (Model CTO-10Avp, Shimadzu). Compounds were analyzed using a CAPCELL PAK C18 column $(\varphi 4.6 \times 150 \mathrm{~mm}$, particle size $3 \mu \mathrm{m}$, Shiseido, Tokyo, Japan), protected by a Nova-Pak C18 $(\varphi 3.9 \times 20 \mathrm{~mm}$, particle size $4 \mu \mathrm{m}$, Waters Co.) guard column, with a mobile phase consisting of $0.7 \%$ sodium perchlorate monohydrate in methanol/ethanol/70\% perchloric acid $(400 / 600 / 1, v / v / v)$ delivered at flow rate $0.6 \mathrm{~mL} / \mathrm{min}$. The peak area was used to quantify UqH-10.

The detection system consisted of a Coulochem II electrochemical detector (ESA, Inc., Chelmsford, MA, USA), connected with two cells. After separation, Uq-10 was reduced to UqH-10 by passing through the Model 5020 guard cell $(-450 \mathrm{mV})$ and was oxidized by passing through the Model 5011 analytical cell (electrode 1, $-100 \mathrm{mV}$; electrode 2, $+350 \mathrm{mV}$ ). The signals of electrode 2 were transmitted to the detector (filter, $10 \mathrm{sec}$; output, $1 \mathrm{~V}$; offset, 20\%; gain range, 0-7 $\mathrm{min}=500 \mathrm{nA}, 7-22 \mathrm{~min}=10 \mathrm{nA}$ ). Chromatographic data were integrated using CLASS-vp (Shimadzu, Japan). UqH-10 and Uq-10 were quantified using linear calibration curves of peak areas versus compound concentrations. Retention times were: UqH-DMG, 4.8 min; UqH-1-DMG, $6.6 \mathrm{~min}$; UqH-4-DMG, $6.7 \mathrm{~min}$; UqH-10, $11.4 \mathrm{~min}$ and $\mathrm{Uq}-10,16.8 \mathrm{~min}$.

\subsection{Dosing Protocol}

We first performed a preliminary experiment to determine the effects of food intake on gastrointestinal absorption of Uq-10 in rats. The contents of commercially available hard capsules (Neuquinon ${ }^{\circledR}$ capsules, Eisai Co., Ltd., Tokyo, Japan) were used as the source of Uq-10. The Uq-10 preparation was suspended in distilled water containing 20\% (w/v) arabic gum. Before the experiment, the fasting group was fasted for $16 \mathrm{~h}$ prior to dosing. In the controlled-feeding group, feeding for $2 \mathrm{~h}$ per day was continued for 3 weeks before dosing and Uq-10 was administered $2 \mathrm{~h}$ after the feeding time. All rats were supplied with drinking water ad libitum. Uq-10 was administered via the esophagus with a gastric tube. Rats were administered $40.5 \mathrm{mmol} / \mathrm{kg} \mathrm{Uq}-10$ in $0.2 \mathrm{~mL} / 100 \mathrm{~g}$ body weight and were given no food after administration. At $0.5,1,2,3,4,6,8$, and $24 \mathrm{~h}$, blood samples $(200 \mu \mathrm{L})$ were collected from the external jugular vein under anesthesia with isoflurane using heparinized syringes. UqH-10 and Uq-10 were extracted as described by Yamashita and Yamamoto [15]. The plasma was isolated by centrifugation, and $50 \mu \mathrm{L}$ plasma aliquots were added to $250 \mu \mathrm{L}$ methanol. After vortex mixing for $30 \mathrm{~s}, 500 \mu \mathrm{L}$ n-hexane was added, followed by vortex mixing for $2 \mathrm{~min}$. The samples were diluted with ethyl acetate and centrifuged at $1250 \times g$ for $3 \mathrm{~min}$. A $5 \mu \mathrm{L}$ aliquot of each organic layer was analyzed by the HPLC system described in Section 3.9.2.

Based on results of this initial experiment, we next treated rats with Uq-10, UqH-4-DMG and UqH-DMG, using the same procedures. UqH-4-DMG and UqH-DMG were suspended in a $20 \%$ propylene glycol aqueous solution. All compounds were administered at $40.5 \mathrm{mmol} / \mathrm{kg}$.

Supplementary Materials: The following are available online, Figure S1. Effects of eserine on enzymatic hydrolysis of UqH-4-DMG by rat and human liver microsomes.

Author Contributions: Conceptualization: S.S., R.H., N.N.-A., and J.T.; methodology: S.S., R.H., and N.N.-A.; validation: S.S. and N.N.-A.; formal analysis: S.S., R.H., and N.N.-A.; investigation: S.S., R.H., N.N.-A., and D.W.; resources: N.N.-A. and J.T.; data curation: S.S. and N.N.-A.; writing—original draft preparation: S.S. and N.N.-A.; writing-review and editing: S.S., R.H., K.M., and J.T.; visualization: S.S. and R.H.; supervision: M.K. and K.M.; project administration: K.M. and J.T.; funding acquisition: Y.K. and J.T. All authors have read and agreed to the published version of the manuscript.

Funding: This research received no external funding.

Acknowledgments: We thank Akihiko Yamasaki and Kentaro Kamata who were a co-researcher and master's student, respectively, in our laboratory that supported the experimental execution of this study.

Conflicts of Interest: The authors declare no conflict of interest. 


\section{References}

1. Ernster, L.; Dallner, G. Biochemical, physiological and medical aspects of ubiquinone function. Biochimica Et Biophysica Acta - Mol. Basis Dis. 1995, 1271, 195-204. [CrossRef]

2. Bhagavan, H.N.; Chopra, R.K. Coenzyme Q10: Absorption, tissue uptake, metabolism and pharmacokinetics. Free Radic. Res. 2006, 40, 445-453. [CrossRef] [PubMed]

3. Hernández-Camacho, J.D.; Bernier, M.; López-Lluch, G.; Navas, P. Coenzyme Q. Front. Physiol. 2018, 9, 44. [CrossRef] [PubMed]

4. $\quad$ Beal, M.F.; Oakes, D.; Shoulson, I.; Henchcliffe, C.; Galpern, W.R.; Haas, R.; Juncos, J.L.; Nutt, J.G.; Voss, T.S.; Ravina, B.; et al. A Randomized Clinical Trial of High-Dosage Coenzyme Q10 in Early Parkinson Disease No Evidence of Benefit. JAMA Neurol. 2014, 71, 543-552. [CrossRef] [PubMed]

5. Yang, Y.K.; Wang, L.P.; Chen, L.; Yao, X.P.; Yang, K.Q.; Gao, L.G.; Zhou, X.L. Coenzyme Q10 treatment of cardiovascular disorders of ageing including heart failure, hypertension and endothelial dysfunction. Clin. Chim. Acta 2015, 450, 83-89. [CrossRef] [PubMed]

6. Hosoe, K.; Kitano, M.; Kishida, H.; Kubo, H.; Fujii, K.; Kitahara, M. Study on safety and bioavailability of ubiquinol (Kaneka QH (TM)) after single and 4-week multiple oral administration to healthy volunteers. Regul. Toxicol. Pharmacol. 2007, 47, 19-28. [CrossRef] [PubMed]

7. Porter, C.J.H.; Trevaskis, N.L.; Charman, W.N. Lipids and lipid-based formulations: optimizing the oral delivery of lipophilic drugs. Nat. Rev. Drug Discov. 2007, 6, 231-248. [CrossRef] [PubMed]

8. Kommuru, T.R.; Gurley, B.; Khan, M.A.; Reddy, I.K. Self-emulsifying drug delivery systems (SEDDS) of coenzyme Q10: formulation development and bioavailability assessment. Int. J. Pharm. 2001, 212, 233-246. [CrossRef]

9. Onoue, S.; Uchida, A.; Kuriyama, K.; Nakamura, T.; Seto, Y.; Kato, M.; Hatanaka, J.; Tanaka, T.; Miyoshi, H.; Yamada, $\mathrm{S}$. Novel solid self-emulsifying drug delivery system of coenzyme $\mathrm{Q}_{10}$ with improved photochemical and pharmacokinetic behaviors. Eur. J. Pharm. Sci. 2012, 46, 492-499. [CrossRef] [PubMed]

10. Cheuk, S.Y.; Shih, F.F.; Champagne, E.T.; Daigle, K.W.; Patindo, J.A.; Mattison, C.P.; Boue, S.M. Nano-encapsulation of coenzyme $\mathrm{Q}(10)$ using octenyl succinic anhydride modified starch. Food Chem. 2015, 174, 585-590. [CrossRef] [PubMed]

11. Sikorska, M.; Lanthier, P.; Miller, H.; Beyers, M.; Sodja, C.; Zurakowski, B.; Gangaraju, S.; Pandey, S.; Sandhu, J.K. Nanomicellar formulation of coenzyme Q10 (Ubisol-Q10) effectively blocks ongoing neurodegeneration in the mouse 1-methyl-4-phenyl-1,2,3,6-tetrahydropyridine model: Potential use as an adjuvant treatment in Parkinson's disease. Neurobiol. Aging 2014, 35, 2329-2346. [CrossRef] [PubMed]

12. Sato, Y.; Yokoyama, S.; Yamaki, Y.; Nishimura, Y.; Miyashita, M.; Maruyama, S.; Takekuma, Y.; Sugawara, M. Enhancement of intestinal absorption of coenzyme Q10 using emulsions containing oleyl polyethylene acetic acids. Eur. J. Pharm. Sci. 2019, 142, 105144. [CrossRef] [PubMed]

13. Takata, J.; Karube, Y.; Hanada, M.; Matsunaga, K.; Matsushima, Y.; Sendo, T.; Aoyama, T. Vitamin-k prodrugs 1. Synthesis of amino-acid esters of menahydroquinone- 4 and enzymatic reconversion to an active form. Pharm. Res. 1995, 12, 18-23. [CrossRef] [PubMed]

14. Takata, J.; Karube, Y.; Hanada, M.; Matsunaga, K.; Matsushima, Y.; Sendo, T.; Oishi, R. Vitamin K prodrugs. 2. Water-soluble prodrugs of menahydroquinone-4 for systemic site-specific delivery. Pharm. Res. 1995, 12, 1973-1979. [CrossRef] [PubMed]

15. Yamashita, S.; Yamamoto, Y. Simultaneous detection of ubiquinol and ubiquinone in human plasma as a marker of oxidative stress. Anal. Biochem. 1997, 250, 66-73. [CrossRef] [PubMed]

Sample Availability: Samples of the compound, UqH-DMG is available from the authors. 